\title{
INFLUÊNCIA DA CONCENTRAÇÃO DE NaOH NA SÍNTESE DE GEOPOLÍMERO COM REAPROVEITAMENTO DE RESÍDUO INDUSTRIAL
}

\author{
Woshington da Silva Brito \\ André Luis Mileo Ferraioli Silva ' \\ Kristoff Svensson ${ }^{2}$ \\ Herbert Pöllmann ${ }^{2}$ \\ Rozineide Aparecida Antunes Boca Santa ${ }^{3}$ \\ Humberto Gracher Riella ${ }^{3}$ \\ José Antônio da Silva Souza ${ }^{4}$
}

\section{Resumo}

A concentração molar do ativador alcalino é um parâmetro importante na síntese de geopolímero com reaproveitamento de cinza volante e metacaulim. $O$ trabalho de pesquisa tem como objetivo avaliar o desempenho mecânico dos geopolímeros com razão de Davidovits $\left(\mathrm{SiO}_{2} / \mathrm{Al}_{2} \mathrm{O}_{3}\right)$ de 2,65, sintetizados com diferentes concentrações molares de $\mathrm{NaOH}\left(5,15,30\right.$ e 40 Molar) no ativador. A reação de geopolimerização foi conduzida a temperatura ambiente de $28^{\circ} \mathrm{C}$ e como ativador utilizou-se hidróxido de sódio $(\mathrm{NaOH})$ e silicato de sódio $\left(\mathrm{Na}_{2} \mathrm{SiO}_{3}\right)$ alcalino 10 Molar. Os geopolímeros sintetizados com 5 e 15 molar de $\mathrm{NaOH}$ apresentaram alta resistência a compressão. Apresentando 40,8 MPa com 28 dias de cura para o geopolímero sintetizado com 15 molar. Os geopolímeros sintetizados com 30 e 40 molar de $\mathrm{NaOH}$ apresentaram baixa resistência a compressão.

Palavras-chave: Geopolímero; $\mathrm{NaOH}$; Cinza leve; Metacaulim.

\section{INFLUENCE OF NaOH CONCENTRATION IN THE SYNTHESIS OF GEOPOLYMER WITH REUSE OF INDUSTRIAL WASTE}

\begin{abstract}
The molar concentration of the alkaline activator is an important parameter in the synthesis of geopolymer with reuse of fly ash waste and metakaolin. The objective of this work was to evaluate the mechanical performance of the Davidovits $\left(\mathrm{SiO}_{2} / \mathrm{Al}_{2} \mathrm{O}_{3}\right)$ geopolymers of 2,65 synthesized with different concentrations of $\mathrm{NaOH}(5,15,30,40$ Molar) in the activator. The geopolymerization reaction was conducted in ambient temperature of $28^{\circ} \mathrm{C}$ and 15 Molar sodium hydroxide $(\mathrm{NaOH})$ and 10 Molar sodium silicate $\left(\mathrm{Na}_{2} \mathrm{SiO}_{3}\right)$, were used as the activator. The geopolymers synthesized with 5 and 15 molar $\mathrm{NaOH}$ showed high compressive strength. Featuring $40.8 \mathrm{MPa}$ with 28 days cure for the 15 molar synthesized geopolymer. The geopolymers synthesized with 30 and 40 molar $\mathrm{NaOH}$ showed low compressive strength. Keywords: Geopolymers; NaOH; Fly ash; Metakaolin.
\end{abstract}

\section{INTRODUÇÃO}

Os geopolímeros são sintetizados a diferentes temperaturas pela ativação alcalina de aluminossilicatos derivados de minerais naturais, argila calcinada ou subprodutos industriais [I]. Geralmente, esta ativação é realizada com metacaulim, cinza volante e silicatos de sódio ou de potássio. Os geopolímeros são ligantes inorgânicos

'Departamento de Engenharia Química - DEQ, Universidade Federal do Pará - UFPA, Belém, PA, Brasil. E-mail:wsbrito3@gmail.com ${ }^{2}$ Department of Geosciences and Geography Martin Luther University Halle-Wittenberg, Deutschland.

${ }^{3}$ Departamento de Engenharia Química, Universidade Federal de Santa Catarina -UFSC, Florianópolis, SC, Brasil.

${ }^{4}$ Programa de Pós-graduação em Engenharia de Recursos Natura da Amazônia - PRODERNA, Universidade Federal do Pará - UFPA, Belém, PA, Brasil.

2176-1523 (C) 2019 Associação Brasileira de Metalurgia, Materiais e Mineração. Publicado pela ABM. Este é um artigo de acesso aberto distribuído sob os termos da licença Creative Commons CC BY-NC-ND (Attribution-NonCommercial-NoDerivs) - https:// creativecommons.org/licenses/by-nc-nd/4.0\%. 
com boa resistência a altas temperaturas e também com boas propriedades mecânicas. Portanto, é uma alternativa atraente ao cimento do tipo Portland, e seu uso permite a reciclagem de grandes quantidades de resíduos industriais. As propriedades mecânicas dos materiais geopoliméricos dependem do cátion alcalino $\left(\mathrm{Na}^{+}\right)$ou $\left(\mathrm{K}^{+}\right)$, da razão de Davidovits $\mathrm{SiO}_{2} / \mathrm{Al}_{2} \mathrm{O}_{3}$ e das condições com que a reação ocorre podendo ser a temperatura ambiente ou utilizando-se câmara térmica com outras temperaturas de processamento do geopolímero [1-3].

Alterar a relação $\mathrm{SiO}_{2} / \mathrm{Al}_{2} \mathrm{O}_{3}$ em geopolímeros permite assim a síntese de materiais com diferentes estruturas. O mecanismo de geopolimerização é particularmente incerto devido à cinética da reação. No entanto, a maioria dos autores concorda que o mecanismo envolve a dissolução, seguido de policondensação de gel [4].

Estudos mostraram que as propriedades mecânicas dos geopolímeros aumentam com a elevação da temperatura de calcinação do caulim para gerar metacaulim, no entanto a temperatura ideal para a calcinação do caulim é de cerca de $700^{\circ} \mathrm{C}$ por 2 horas $[5,6]$.

O desenvolvimento de geopolímero a base de cinza proveniente da queima de carvão vem se mostrando promissor pelo reaproveitamento desse resíduo. A demanda global por carvão crescerá até 2030, atingindo uma duplicação em relação à demanda atual. Com o crescente consumo de carvão para a geração de energia, haverá uma produção aumentada de cinzas como um subproduto da combustão do carvão [7-9].

A ativação alcalina de resíduos, especialmente aqueles oriundos de atividades industriais ou de mineração que possuem em sua composição os aluminossilicatos, tem se tornado uma importante área de pesquisa. $O$ atrativo é a possibilidade de se usar esses materiais para sintetizar cimentos economicamente mais viáveis e ecologicamente corretos, dessa forma abrem-se novas oportunidades para a produção de cimentos geopoliméricos especiais com propriedades diferentes daquelas apresentadas pelo Portland comum [I0].

\section{MATERIAIS E MÉTODOS}

O resíduo cinza volante foi coletado no overflow (saída) do ciclone das caldeiras de leito fluidizado tipo circulante em condições de $900^{\circ} \mathrm{C}$ de temperatura e $120 \mathrm{KPa}$ de pressão. Esse resíduo foi utilizado como fonte de Si e Al na síntese de geopolímero. Além do cinza volante foi utilizado também como fonte de Si e Al o metacaulim produzido pela calcinação de caulim em temperatura de $800^{\circ} \mathrm{C}$ por duas horas.

Para as análises da estrutura cristalina da cinza, do caulim e do metacaulim foi utilizado difratometro de raios $X(D R X)$ de marca Bruker LyuxEye. A Cinza e o metacaulim foram submetidos a analise granulométrica de suas partículas, em granulometro a laser de marca Fritsch Analysette 22 Microtec plus. Foi formulado geopolímero a base de cinza volante e metacaulim com razão de 2,65 de $\mathrm{SiO}_{2} / \mathrm{Al}_{2} \mathrm{O}_{3}$. A Tabela I apresenta a composição do geopolímero estudado com sua respectiva razão de Davidovits $\left(\mathrm{SiO}_{2} / \mathrm{Al}_{2} \mathrm{O}_{3}\right)$.

$O$ ativador alcalino usado na síntese do geopolímero foi composto de uma solução de hidróxido de sódio $(\mathrm{NaOH})$ micro perola (Neon, $97 \%$ pureza). Também se usou solução de silicato de sódio alcalino $\left(\mathrm{Na}_{2} \mathrm{SiO}_{3}\right)$ (Manchester Química do Brasil S.A., $\mathrm{SiO}_{2} / \mathrm{Na}_{2} \mathrm{O}=3,2$ ). A Tabela 2 apresenta a composição do ativador.

As composições foram preparadas em misturador mecânico e conformadas em moldes cilíndricos de $100 \mathrm{~mm}$ de altura e diâmetro de $50 \mathrm{~mm}$. Após a moldagem os moldes foram submetidos à cura a temperatura ambiente de $28^{\circ} \mathrm{C}$. Os geopolímeros com 24 horas(I dia), 7, 28 e 60 dias de cura foram submetidos a ensaio de resistência a compressão em prensa Emic SSII300.

\section{RESULTADOS E DISCUSSÃO}

A Figura I apresenta o resultado do ensaio do DRX da cinza volante, do caulim e do metacaulim calcinado a $800^{\circ} \mathrm{C}$ por 2 horas.

Tabela I. Composição formulada entre a cinza volante e o metacaulim para obtenção do geopolímero

\begin{tabular}{cccc}
\hline Geopolímero & Cinza volante (\%) & Metacaulim (\%) & $\mathbf{S i O}_{2} / \mathbf{A l}_{2} \mathbf{O}_{3}$ \\
\hline 01 & 42,31 & 57,69 & 2,65 \\
\hline
\end{tabular}

\% - Em massa.

Tabela 2. Concentração molar e proporções da solução ativadora

\begin{tabular}{|c|c|c|}
\hline Ativador: & $\mathrm{NaOH}$ & $\mathrm{Na}_{2} \mathrm{SiO}_{3}$ \\
\hline \multirow[t]{4}{*}{ Concentração Molar: } & 5 & 10 \\
\hline & 15 & 10 \\
\hline & 30 & 10 \\
\hline & 40 & 10 \\
\hline Proporção ( $\mathrm{NaOH}: \mathrm{Na}_{2} \mathrm{SiO}_{3}$ ): & I:3 em massa de solução & \\
\hline
\end{tabular}



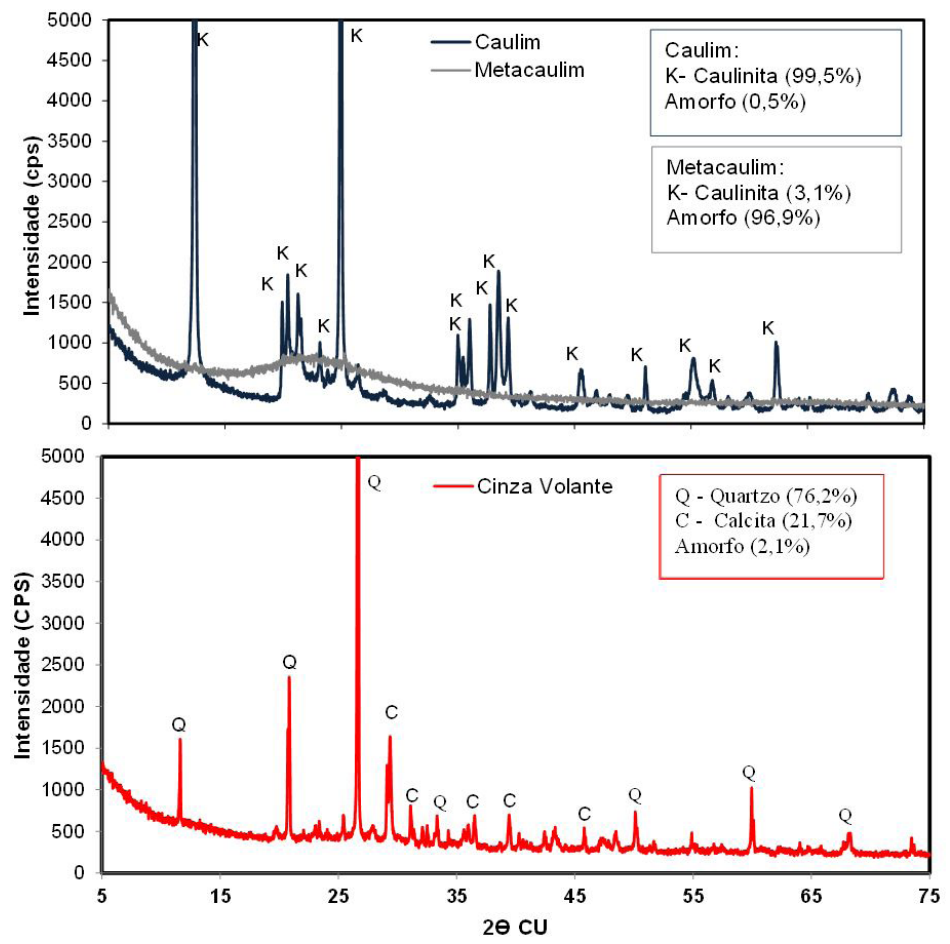

Figura I. DRX do caulim, do metacaulim e da cinza volante.

Conforme demonstrado na Figura I, a calcinação do caulim a $800^{\circ} \mathrm{C}$ por duas horas foi satisfatória, pois gerou uma mudança na fase cristalina transformando-a em fase amorfa atingindo um teor amorfo de $(96,9 \%)$ de acordo com o método de Rietveld, caracterizando o metacaulim como um material reativo para a reação de geopolimerização. A mudança ocorrida no material após o tratamento térmico libera Al e o Si para reação de geopolimerização. Caulim calcinado numa faixa entre $600^{\circ} \mathrm{C} \mathrm{e} 800^{\circ} \mathrm{C}$ por um intervalo de tempo entre 2 e 4 horas, vem se mostrando adequado na obtenção de metacaulim aplicado ao desenvolvimento de geopolímero [II,12].

A cinza volante gerada nas refinarias de alumínio apresenta quartzo $(72,2 \%)$ e calcita $(21,7 \%)$ em sua constituição como evidenciado no difratograma da cinza na Figura I. A distribuição de tamanho de partícula das materiais primas usadas na síntese do geopolímero resultou em um diâmetro médio $\left(\mathrm{d}_{50}\right)$ de $24 \mu \mathrm{m}$ para a cinza volante e de $88 \mu \mathrm{m}$ para o metacaulim.

A figura 2 apresenta o resultado do ensaio de resistência a compressão em MPa para o geopolímero com 7 e 28 dias de cura sintetizado com concentrações de $5,15,30$ e 40 molares de $\mathrm{NaOH}$ no ativador.

Pela figura 2 o geopolímero sintetizado com 5 e 15 molares de $\mathrm{NaOH}$ apresentaram alta resistência a compressão, atingindo valores de 29,68 e 40,08 $\mathrm{MPa}$ respectivamente com 28 dias de cura a temperatura ambiente. Os geopolímeros sintetizados com 30 e 40 molares de $\mathrm{NaOH}$ apresentaram baixa resistência a compressão em comparação aos geopolímeros sintetizados a 5 e 15 molares.

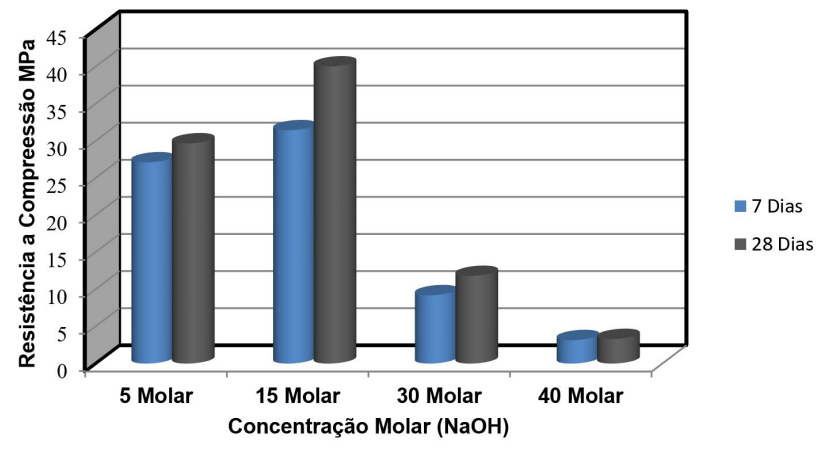

Figura 2. Concentração molar de $\mathrm{NaOH}$ com 7 e 28 dias de cura.

O excesso de hidróxido de sódio, além de causar carbonatação, diminui a resistência mecânica, aumenta a porosidade e também ocorre uma imediata reação de geopolimerização, ocasionando em uma rápida cura [13]. A figura 3 apresenta o resultado de ensaio de resistência a compressão em MPa para o geopolímero sintetizado a 15 molar de $\mathrm{NaOH}$ para 24 horas, 7, 28 e 60 dias de cura a temperatura ambiente.

De acordo com o resultado da figura 3 o geopolímero atingiu em 24horas de cura a temperatura ambiente resistência igual à resistência de concreto convencional com 28 dias de cura a base de cimento portland.

A figura 4 apresenta o EDS da estrutura do geopolímero sintetizado com 15 molar de $\mathrm{NaOH}$ após sofrer o ensaio de resistência a compressão aos 28 dias de cura. 
Pela figura 4 observa-se uma morfologia densa e uniforme do geopolímero sintetizado com 15 molar de $\mathrm{NaOH}$ o que lhe confere alta resistência a compressão. Salehi [14] constatou em seus estudos que o aspecto morfológico mais denso e uniforme confere maior desempenho mecânico aos geopolímeros e consequentemente maior avanço de reação de geopolimerização.

No espectro do EDS da figura 4 a presença do Al e do Si enfatiza o caráter alumínio silicato da reação de geopolimerização. A presença do $\mathrm{Na}$ no espectro é devido ao ativador a base de hidróxido de sódio.
A figura 5 evidencia a morfologia uniforme e densa através da técnica de Microscopia eletrônica por varredura (MEV) do geopolímero sintetizado com I 5 molar de $\mathrm{NaOH}$, nas ampliações 500, 1500 e 2000 X respectivamente.

De acordo com a figura 5 , observa-se a formação de zonas na forma de placas bem definidas indicando uma morfologia uniforme servindo como parâmetro indicativo que a síntese nas condições 15 molar resulta em alta resistência a compressão. As fissuras entre as placas densas e uniformes é devido a microscopia ter sido realizada após o ensaio de compressão.

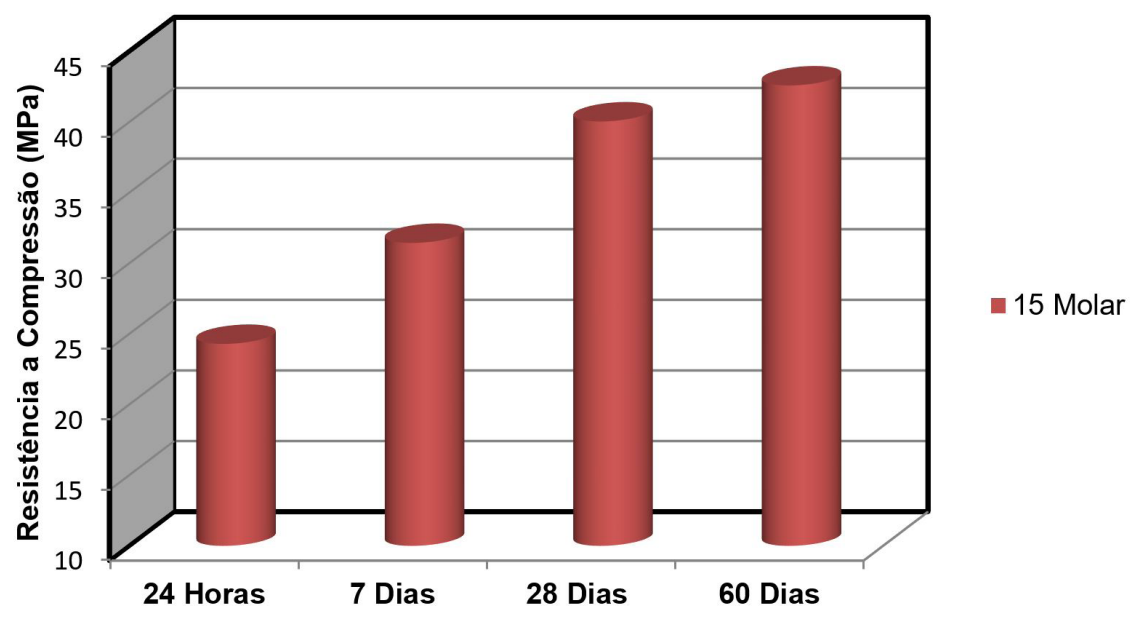

Figura 3. Geopolímero sintetizado com I5 Molar de $\mathrm{NaOH}$ com diferentes tempos de cura.

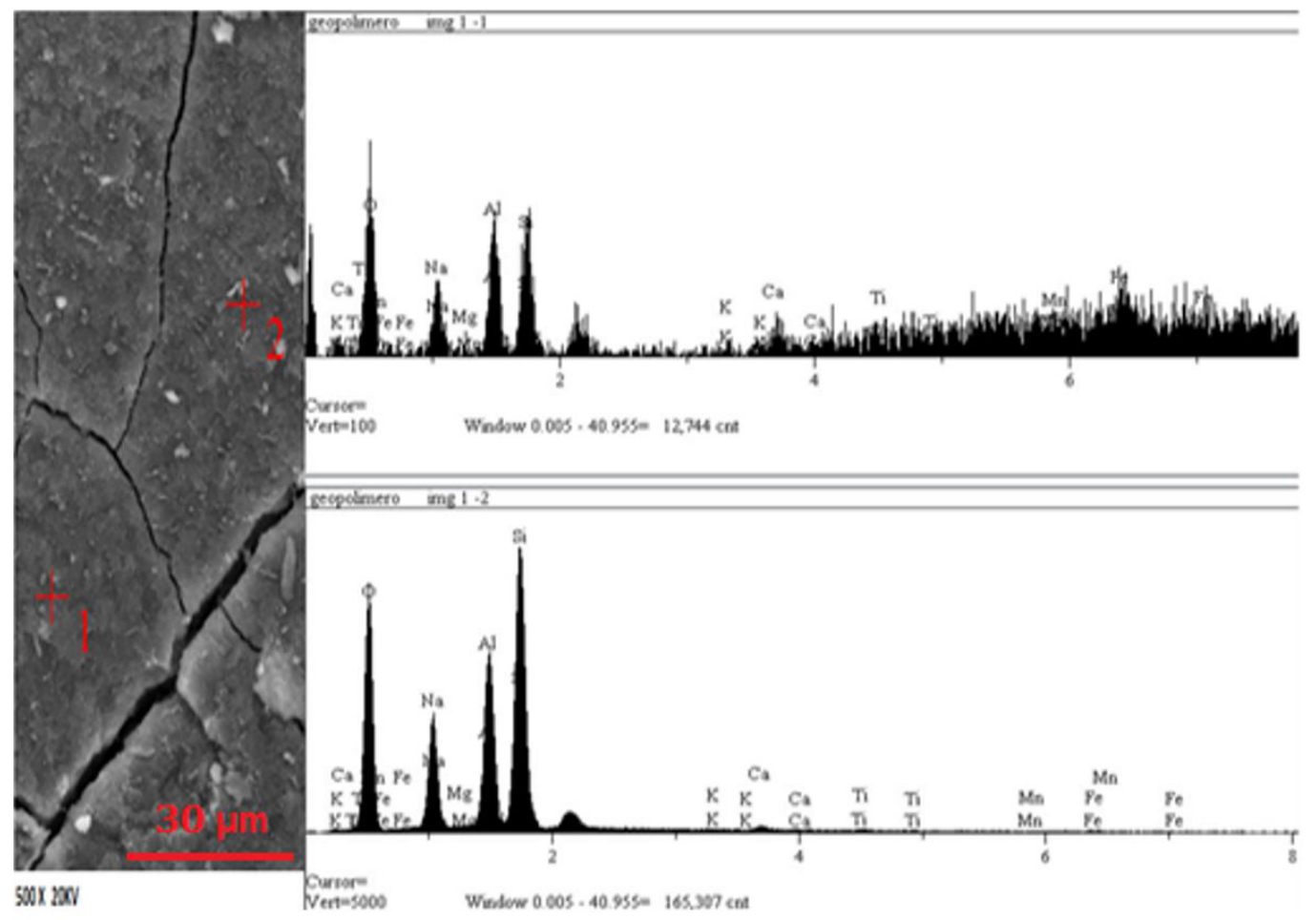

Figura 4. EDS do Geopolímero sintetizado com 15 molar de $\mathrm{NaOH}$. 

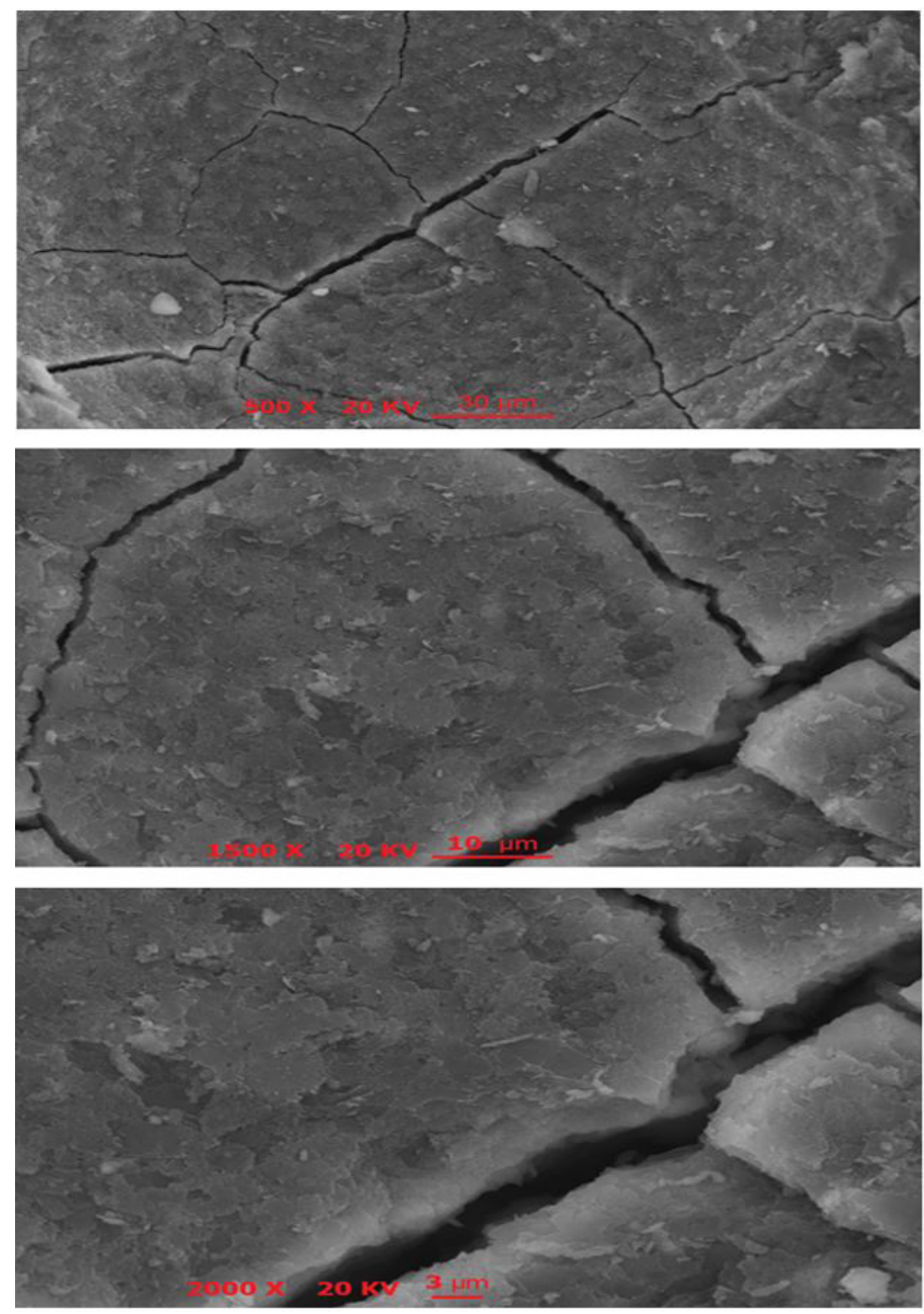

Figura 5. Microscopia eletrônica por varredura (MEV) do geopolímero sintetizado com I 5 molar de NaOH nas ampliações 500, I 500 e $2000 X$.

\section{CONCLUSÂO}

Pela pesquisa conclui-se que o geopolímero a base de metacaulim e cinza volante com razão de 2,65 de $\mathrm{SiO}_{2} / \mathrm{Al}_{2} \mathrm{O}_{3}$ apresentou melhor resistência a compressão quando sintetizado com $\mathrm{NaOH}$ em concentração (5M e 15 molares. Concentrações de 30 e 40 molares interferiram na reação gerando geopolímeros com baixa resistência a compressão. O geopolímero sintetizado a temperatura ambiente com ativador em composição de 15 molar de hidróxido de sódio e 10 molares de silicato de sódio alcalino apresentou-se como uma alternativa para suprir a demanda na área cimenticia, atingindo resistência superior a resistência de concretos convencionais a base de cimento portland. Além disso, os geopolímeros foram produzidos com baixa emissão de $\mathrm{CO}_{2}$, quando comparado aos cimentos convencionais, o que contribui com o meio ambiente. A pesquisa conclui que os geopolímeros sintetizados possuem grande potencial para a produção de materiais geopoliméricos.

\section{Agradecimentos}

Os autores agradecem a CAPES, CNPq, PRODERNA, UFPA e a empresa HYDRO ALUNORTE pelo incentivo a pesquisa. 


\section{REFERENCIAS}

I Davidovits, J. Geopolymer Chemistry e Aplication. 2. ed. Saint-Quentin: Institute Geopolymere; Polymers and Geopolymers. 2008; p.9

2 Duxson P, Provis JL, Lukey GC, Mallicoat SW, Kriven WM, Deventer JSJ. Understanding the relationship between geopolymer composition, microstructure and mechanical properties. Colloids and Surfaces. A, Physicochemical and Engineering Aspects. 2005;269:47-58.

3 Salih MA, Abang A A A, Farzadnia N. Characterization of mechanical and microstructural properties of palm oil fuel ash geopolymer cement paste. Construction \& Building Materials. 2014;65:592-603.

4 Duxson P, et al. Geopolymer technology: the current state of the art. Journal of Materials Science. 2007;42(9):29I72933.

5 Wang MR, Jia DC, He PG, Zhou Y, Lett M. Influence of calcination temperature of kaolin on the structure and properties of final geopolymer. Materials Letters. 2010;64:255I-2554.

6 Cioffi R, Maffucci L, Santoro L. Optimization of geopolymer synthesis by calcination and polycondensation of a kaolinitic residue. Resources, Conservation and Recycling. 2003;40:27-38.

7 Castro GJP, Silva AP, Cano RP, Durán SJ, Albuquerque A. Potential for reuse of tungsten mining waste-rock in technical-artistic value added products. Journal of Cleaner Production. 20I2;25:34-4I.

8 Sakulich AR. Reinforced geopolymer composites for enhanced material greenness and durability. Sustainable Cities and Society. 201 I; I:195-210.

9 Karthik A, Sudalaimani K, Vijayakumar CT. Durability study on coal fly ash-blast furnace slag geopolymer concretes with bio-additives. Ceramics International. 2017;43:1 I935-II 943.

10 Zhang J, He Y, Wang YP, Mao J, Cui XM. Synthesis of a selfsupporting faujasite zeolite membrane using geopolymer gel for separation of alcohol/water mixture. Materials Letters. 2014;116:167-I70.

II Davidovits J. Geopolymers based on natural and synthetic metakaolin - A critical review. New York: Elsevier; 2015.

12 Medri V, Fabbri S, Dedecek J, Sobalik Z, Tvaruzkova Z, Vaccari A. Role of the morphology and the dehydroxylation of metakaolins on geopolymerization. Applied Clay Science. 2010;50:538-545.

13 Narayanan A. An Experimental Investigation on Flyash-based Geopolymer Mortarunder different curing regime for Thermal Analysis. Energy and Buildings. 2017; I38:539-545.

I4 Salehi S, Khattak MJ, Bwala AH, Karbalaei FS. Characterization, morphology and shear bond strength analysis of geopolymers: Implications for oil and gas well cementing applications. Journal of Natural Gas Science and Engineering. 2017;38:323-332.

Recebido em: 21 Jul. 2017

Aceito em: I I Jul. 2018 